\title{
Structure and Deformation Mechanism of the Quasicrystal Strengthening Phase in
} Al-Mn-Be-Cu Alloys

\author{
James Ciston $^{1}$, Colin Ophus ${ }^{1}$ and Boštjan Markoli ${ }^{2}$ \\ 1. National Center for Electron Microscopy, Lawrence Berkeley National Laboratory, Berkeley, \\ California, USA \\ 2. Faculty of Natural Sciences and Engineering, University of Ljubljana, Ljubljana, Slovenia
}

Quasicrystals are a highly unusual class of materials that have received increasing attention [1]. While much is known about the fundamental structures and behaviors of these materials, the engineering applications have proven limited. It has been recently shown that aluminum alloys containing $\mathrm{Mn}, \mathrm{Be}$, and $\mathrm{Cu}$ can be reinforced through the precipitation of quasicrystals at cooling rates as low as $100 \mathrm{~K} / \mathrm{sec}$, which enables near-final shape casting into water-cooled dies [2]. Due to a lack of long-range periodicity, the embedded quasicrystal phase impedes the motion of matrix dislocations during deformation, leading to yield strengths over $450 \mathrm{MPa}$ without additional heat treatment of the as-cast material. These quasicrystal-strengthened alloys also exhibit very large failure strains of $90 \%$ in compression and $40 \%$ in tension indicating superplastic behavior [3], which is a major advantage over typical hard but brittle intermetallic strengthening phases in aluminum alloys.

Using the TEAM 0.5 aberration-corrected high resolution scanning transmission electron microscope (STEM) at NCEM, we have investigated the structure of both as-cast and deformed $\mathrm{Al}_{94} \mathrm{Mn}_{2} \mathrm{Be}_{2} \mathrm{Cu}_{2}$ alloys containing 50-200nm icosahedral-phase quasicrystalline precipitates to elucidate the deformation mechanism governing the observed superplastic strain. High angle annular dark field (HAADF) STEM and parallel beam diffraction patterns were recorded on the 2-, 3-, and 5-fold axes of a single quasicrystalline precipitate in the as-cast material, confirming the primitive icosahedral structure. After ex-situ deformation at room temperature, precipitates are observed with planar defects that are incompatible with a conventional quasicrystal structure. Dark field imaging confirms that the deformed particles are multiply-twinned, and present a pseudo 5-fold diffraction pattern (Fig 2). Both the crystalline structure and phason defects observed in precipitate particles by HAADF STEM after deformation are strikingly similar to the defects in deformed T-phase Al-Mn-Pd complex metal alloy [4]. The origin of superplastic deformation in quasicrystal-strengthened alloys may be related to a martensitic-like transformation from a true quasicrystalline i-phase to a complex metal alloy structure, consistent with the large energy dissipation required at large strains. [5] 

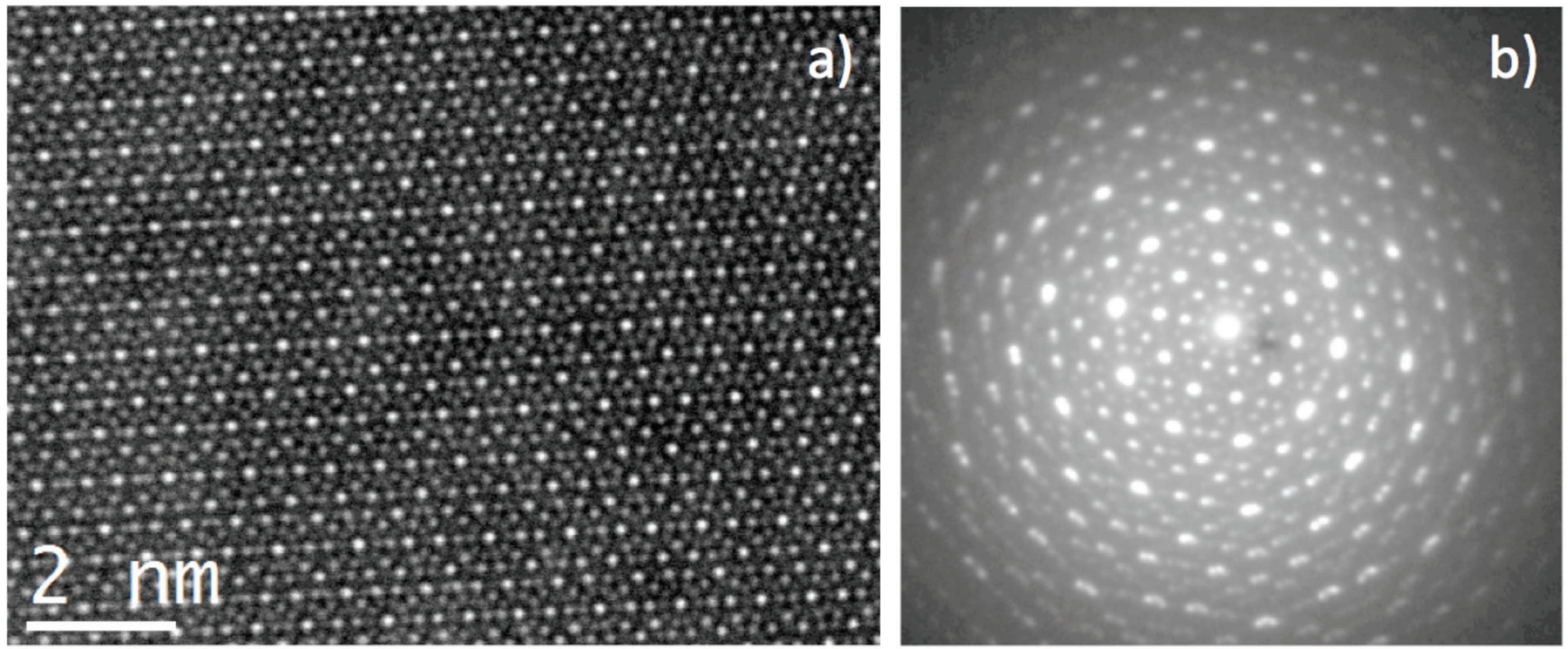

Figure 1. a) Aberration-corrected HAADF STEM image of an undeformed Al-Mn-Cu-Be i-phase quasicrystal precipitate along the 5-fold symmetry projection (Wiener filtered) b) Electron diffraction pattern of the same particle along the 5 -fold projection axis
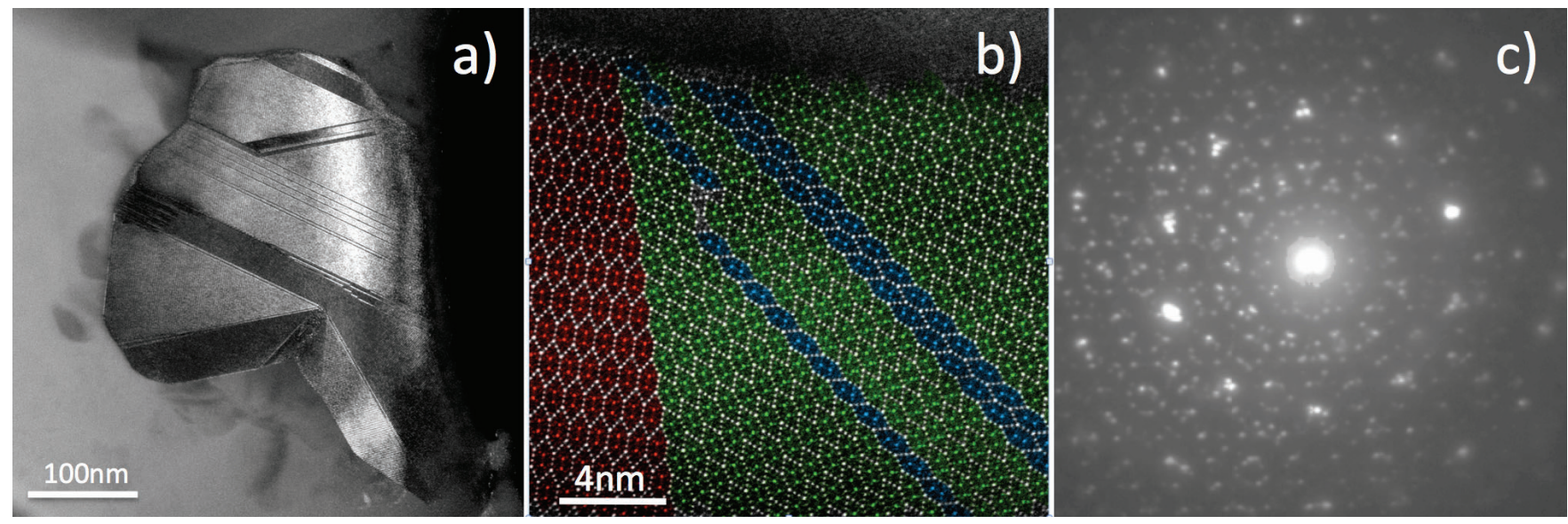

Figure 2. a) Dark field image of an ex-situ deformed embedded quasicrystal precipitate indicating planar defects b) Aberration-corrected HAADF STEM image of an ex-situ deformed precipitate color coded according to the local domain orientation c) Electron diffraction pattern of the same particle indicating the presence of a pseudo-5-fold symmetry

References:

[1] D Shechtman, et al, Physical Review Letters, 53 (1984) p. 1951.

[2] F Zupanic, et al, J. Alloys and Compounds, 452 (2008) p. 343.

[3] B Markoli, et al, Materialwissenschaft und Werkstofftechnik, 43 (2012), p. 340.

[4] M Heggen, et al, Nature Materials, 9 (2010) p. 332.

[5] This work was performed at the National Center for Electron Microscopy, which is supported by the Office of Basic Energy Sciences of the U.S. Department of Energy under Contract No.

DE-AC0205CH11231. 